\title{
Effect of forage availability and sward phenological stage on daily grazing time of ewes
}

\author{
C. Roguet, S. Prache, M. Petit
}

\section{Laboratoire adaptation des herbivores aux milieux, Inra, 63122 Saint-Genès-Champanelle, France}

This study analyses how ewes modify their daily grazing time when intake rate decreases due to forage depletion or sward heterogeneity. Eleven dry ewes grazed four sward structures successively under rotational grazing: M (flowerhead appearance); R (full flowerhead emergence); V1 (summer vegetative); and V2 (autumn vegetative). Daily grazing time (DGT) was assessed twice a week, from dawn to dusk, from automated photographs taken every $3 \mathrm{~min}$. GT was defined as the time spent head down. Green leaf mass (GLM) decreased curvilinearly with time from $0.83,1.42,1.37$, $0.65 \mathrm{tDM} \cdot \mathrm{ha}^{-1}$ to $0.09,0.10,0.13$ and $0.20 \mathrm{tDM} \cdot \mathrm{ha}^{-1}$, for $\mathrm{M}, \mathrm{R}, \mathrm{V} 1$ and $\mathrm{V} 2$, respectively. Above $0.2 \mathrm{tDM} \cdot \mathrm{ha}^{-1}$, DGT increased linearly with GLM depletion from $259,313,298,352$ to 517,608 , 456,521 min for $M, R, V 1$ and $V 2$, respectively $\left(R^{2}=0.80\right.$ to $\left.0.95 \%\right)$. Regressions for $M, R$ and $\mathrm{V} 1+\mathrm{V} 2$ had the same intercept $(573 \mathrm{~min}, P=0.31)$ but the slope was slower $(P=0.007)$ for $\mathrm{R}(-0.183)$ than for $\mathrm{V} 1+\mathrm{V} 2(-0.258)$ and $\mathrm{M}(-0.363)$. Below $0.2 \mathrm{tDM} \cdot \mathrm{ha}^{-1}$, DGT dropped sharply to 393 (M), 506 (R) and 300 (V1) min. DGT was not significantly affected by weather, although the shortest DGTs onV1 were observed on very hot days $\left(\operatorname{Tmax}>25^{\circ} \mathrm{C}\right)$. For each structure, DGT varied significantly between individuals $(P<0.05)$, animals ranking in the same order whatever the GLM. DGT was confirmed to be a compensation adjustement to the decrease in intake rate when forage availability goes down or when sward is heterogeneous.

\section{Préférences alimentaires des brebis et quantités ingérées}

\section{G. Béchet}

Laboratoire adaptation des herbivores aux milieux, Inra, 63122 Saint-Genès-Champanelle, France

Douze brebis limousines taries ont été réparties en trois groupes (I, II, III) différant par la teneur croissante en azote de leurs fèces au pâturage. Elles ont ensuite pâturé durant 11 j une parcelle de dactyle partiellement épiée ( $40 \%$ des contacts de surface au stick). Les teneurs moyennes en azote fécal ont de nouveau été significativement $(p<0,05)$ différentes : $1,58^{\mathrm{a}} ; 1,74^{\mathrm{ab}}$ et $2,06^{\mathrm{b} \%}$ de la MO pour I, II et III, ce qui correspond à des digestibilités de la MO estimées à 0,$57 ; 0,60$ et 0,65 . De même, les teneurs fécales en alcanes ont également été différentes pour le C33:140, $175^{\mathrm{ab}}$ et $230^{\mathrm{b}} \mathrm{mg} \cdot \mathrm{kg}^{-1}$ de MO respectivement. Cela résulte sans doute d'une préférence croissante de I à III pour les feuilles de dactyle à la fois plus riches en alcanes que les tiges et plus digestibles. Les quantités ingérées ont été calculées à l'aide de la méthode des alcanes sur l'ensemble du séjour sur la parcelle, la teneur en alcanes de l'ingéré étant estimée par un même prélèvement manuel pour toutes les brebis. Elles ont diminué significativement $(p<0,01)$ de $22,6^{\mathrm{a}} \mathrm{gMS} \cdot \mathrm{kg}^{-1}$ poids vif à $17,7^{\mathrm{b}} \mathrm{g}$ et $12,0^{\mathrm{c}} \mathrm{g}$ de I à III. Ces valeurs correspondent à 11,$5 ; 9,5$ et 7,0 gMOD $/ \mathrm{kg}$ PV. Ces écarts d'ingestion sont en fait sous-estimés en ne tenant pas compte des différences de choix entre les groupes de brebis. Une préférence marquée pour les feuilles pourrait ainsi limiter l'ingestion, surtout en fin de parcelle. 